\title{
Two Analytical Methods for Detection and Elimination of the Static Hazard in Combinational Logic Circuits
}

\author{
Mihai Grigore Timis, Alexandru Valachi, Alexandru Barleanu, Andrei Stan \\ Automatic Control and Computer Engineering Faculty, Technical University Gh.Asachi, Iasi, Romania \\ Email:mtimis@tuiasi.ro, avalachi@tuiasi.ro, abarleanu@tuiasi.ro, andreis@tuiasi.ro
}

Received August 18, 2013; revised September 18, 2013; accepted September 26, 2013

Copyright (C 2013 Mihai Grigore Timis et al. This is an open access article distributed under the Creative Commons Attribution License, which permits unrestricted use, distribution, and reproduction in any medium, provided the original work is properly cited.

\begin{abstract}
In this paper, the authors continue the researches described in [1], that consists in a comparative study of two methods to eliminate the static hazard from logical functions, by using the form of Product of Sums (POS), static hazard "0". In the first method, it used the consensus theorem to determine the cover term that is equal with the product of the two residual implicants, and in the second method it resolved a Boolean equation system. The authors observed that in the second method the digital hazard can be earlier detected. If the Boolean equation system is incompatible (doesn't have solutions), the considered logical function doesn't have the static 1 hazard regarding the coupled variable. Using the logical computations, this method permits to determine the needed transitions to eliminate the digital hazard.
\end{abstract}

Keywords: Combinational Circuits; Static Hazard; Logic Design; Boolean Functions

\section{Introduction}

Under certain conditions, on the output of the logical signals may occur unwanted transitions. These transitions are known as glitches. The logic glitch is a kind of unwanted noise presenting inthe output signal that can initiate an uncontrollable process. In the next level there is an input signal [2].

We can distinguish three types of noise that is introduced in CLC (Combinational Logic Circuits), called hazards (Static, Dynamic and Function Hazards).

In the following we consider only the static hazard problem in combinational logic systems, called static hazard " 0 ".

- Static 1 hazard, also called SOP (Sum of Products) hazard —a glitch that occurs in otherwise steady-state 1 output signal from SOP logic;

- Static 0 hazard, also called POS (Product of Sums) hazard-a glitch that occurs in otherwise steady-state 0 output signal from POS logic.

Static Hazards in Two-Level Combinational Logic Circuits (Consensus Method [2]).

We will initially define:

- Coupled variable; a variable input is complemented within a term of function and uncomplemented in another term of the same function.
- Coupled term; one of two terms containing only one coupled variable.

- Residue; the part of a coupled term that remains after removing the coupled variable.

- Hazard cover (or consensus term).

The RPI (Redundant Prime Implicant) required to eliminate the static hazards:

AND the residues of coupled p-term to obtain the SOP hazard cover,

OR the residues of coupled s-term to obtain the POS hazard cover.

POS example: any logic function can be described as:

$$
y=\left(e_{0}+x_{i}\right)\left(e_{1}+\overline{x_{i}}\right)
$$

where

$$
\begin{aligned}
& e_{0}=y\left(x_{n-1}, x_{n-2}, \cdots, x_{i+1}, 0, x_{i-1}, \cdots, x_{0}\right) \\
& e_{1}=y\left(x_{n-1}, x_{n-2}, \cdots, x_{i+1}, 1, x_{i-1}, \cdots, x_{0}\right)
\end{aligned}
$$

Sometimes, the same function can be describes as:

$$
y=\left(a+x_{i}\right)\left(b+\overline{x_{i}}\right) c
$$

Using the (1) form, we can say:

$$
\begin{aligned}
& e_{0}=a c \\
& e_{1}=b c
\end{aligned}
$$


Using the algorithm described in [3], if $c \leq a+b$, the expression from (2), (2.1) doesn't present static hazard in relation with the $x_{i}$ input, and if $a=b=0$, then results $c=0$.

The condition to have static hazard in relation with the $x_{i}$ input, is when $a=b=0$ and $c=1$.

The consensus method [4] consists of determination of coupled terms, then by removing the coupled variables we obtain residual values.

That meaning the (2) equation can be written like:

$$
y=\left(a+x_{i}\right)\left(b+\overline{x_{i}}\right) c(a+b)
$$

It can be observed that the expression of the function is multiplied by the sum of residual values, the new expression presents static hazard in relation with the $x_{i}$ input.

We proposed as example the 4 inputs logic function:

$$
\begin{aligned}
y= & y\left(x_{4}, x_{3}, x_{2}, x_{1}, x_{0}\right) \\
= & R_{0}(1,3,6,7,9,11,14,17,19,20,25,27,28) \\
& +R_{\Phi}(0,5,10,16,23,24,29,31)
\end{aligned}
$$

Using the Quine-McCluskey minimization method we obtain the equation from (5) and also the residual values determined by $x_{0}$ input:

$$
\begin{aligned}
& y=\left(x_{4}+x_{3}+\overline{x_{2}}+\overline{x_{1}}\right)\left(\overline{x_{4}}+x_{1}+x_{0}\right)\left(x_{2}+\overline{x_{0}}\right) \\
& \cdot\left(x_{4}++\overline{x_{2}}+\overline{x_{1}}+x_{0}\right) \\
& a=\left(\overline{x_{4}}+x_{1}\right)\left(x_{4}+\overline{x_{2}}+\overline{x_{1}} x_{0}\right) \\
& b=x_{2} \\
& a+b=x_{2}+\overline{x_{4}} \overline{x_{2}}+\overline{x_{4}} \overline{x_{1}} x_{0}+x_{4} x_{1}+\overline{x_{2}} x_{1}=x_{2}+\overline{x_{4}}+x_{1}
\end{aligned}
$$

The expression of no static hazard in relation with $x_{0}$ input:

$$
\begin{aligned}
y= & \left(x_{4}+x_{3}+\overline{x_{2}}+\overline{x_{1}}\right)\left(\overline{x_{4}}+x_{1}+x_{0}\right)\left(x_{2}+\overline{x_{0}}\right) \\
& \cdot\left(x_{4}+\overline{x_{2}}++\overline{x_{1}}+x_{0}\right)\left(\overline{x_{4}}+x_{2}+x_{1}\right)
\end{aligned}
$$

\section{Method of Resolving of Boolean Equations [5]}

In this paragraph we apply the consensus method [5] and the method of solving some specific Boolean equations.

If $y=\left(a+x_{i}\right)\left(b+\bar{x}_{i}\right) c$, by resolving the next system equations it can be determined the vectors input values which presents static hazard.

$$
\begin{aligned}
a & =0 \\
b & =0 \\
c & =1
\end{aligned}
$$

If the (7) system has no solution, the function doesn't presents static hazard in relation with $x_{i}$.

Therefore, the expression of the function becomes:

$$
\begin{aligned}
& a=\left(\overline{x_{4}}+x_{1}\right)\left(x_{4}+\overline{x_{2}}+\overline{x_{1}}+x_{0}\right)=0 \\
& b=x_{2}=0 \\
& c=x_{4}+x_{3}+\overline{x_{2}}+\overline{x_{1}}=1
\end{aligned}
$$

Therefore, $x_{2}=0$ imposes the reduction of the system to: $\overline{x_{4}}+x_{1}=0$ or $x_{4} \overline{x_{1}}=1$

So, the solution is:

$$
\begin{aligned}
& x_{4}=1 \\
& x_{3}=\Phi \\
& x_{2}=0 \\
& x_{1}=0
\end{aligned}
$$

So, the function will have hazard at commutation

$$
\begin{aligned}
& x_{4} X_{3} X_{2} X_{1} X_{0} \\
& 10000 \leftrightarrow 10001(16-17) \\
& 11000 \leftrightarrow 11001 \quad(24-25)
\end{aligned}
$$

So, in the POS relation will be added the multiplied prime implicant $\overline{x_{4}}+x_{2}+x_{1}$.

The function will have the same expression like in (7).

\section{Static Hazards in Two-Level Combinational Logic Circuits}

We will consider two analytical methods to detect and eliminate this type of hazard:

(A) Consensus method [1]

We will initially define:

- Coupled variable; a variable input is complemented within a term of function and uncomplemented in another term of the same function.

- Coupled term; one of two terms containing only one coupled variable.

- Residue; the part of a coupled term that remains after removing the coupled variable.

- Hazard cover (or consensus term).

The RPI (Redundant Prime Implicant) required to eliminate the static hazards:

- AND the residues of coupled p-term to obtain the SOP hazard cover,

- OR the residues of coupled s-term to obtain the POS hazard cover.

Example 1. Lets consider the logic function $f\left(x_{2} x_{1} x_{0}\right)=R_{1}(2,3,5,7)$.

a) SOP example: will be determined the prime implicants using Veitch-Karnaugh or Quine-McCluskey methods, as:

$$
\begin{aligned}
& A=\overline{x_{2}} \cdot x_{1} \\
& B=x_{1} \cdot x_{0} \\
& C=x_{2} \cdot x_{0}
\end{aligned}
$$

One of the minimal equations is: 


$$
y=A+C=\overline{x_{2}} \cdot x_{1}+x_{2} \cdot x_{0}
$$

where we have:

- coupled variable: $x_{2}$

- coupled terms: $x_{2} \cdot x_{1}, x_{2} \cdot x_{0}$

- residues: $x_{1}, x_{0}$

- consensus term: $x_{1} \cdot x_{0}$

Therefore, the logic expression that has no static hazard in relation to $x_{2}$ variable is:

$$
y=\overline{x_{2}} \cdot x_{1}+x_{2} \cdot x_{0}+x_{1} \cdot x_{0}
$$

b) POS example: will be determined the prime implicants using Veitch-Karnaugh or Quine-Mc Cluskey methods, as:

$$
\begin{aligned}
& a=x_{2}+x_{1} \\
& b=x_{1}+x_{0} \\
& c=\overline{x_{2}}+x_{0}
\end{aligned}
$$

One of the minimal equations is:

$$
y=\left(x_{2}+x_{1}\right) \cdot\left(\overline{x_{2}}+x_{0}\right) \cdot\left(x_{1}+x_{0}\right)
$$

where we have:

- coupled variable: $x_{2}$

- coupled terms: $x_{2}+x_{0}, x_{2}+x_{1}$

- residues: $x_{1}, x_{0}$

- consensus term: $x_{1}+x_{0}$

The equation (13) shows no static 0 hazard.

Example 2. Let's consider the function of four variables $y=f\left(x_{3} x_{2} x_{1} x_{0}\right)=R_{1}(0,1,2,5,6,7,8,9,10,14)$.

SOP hazard: will be determined the prime implicants using Quine-McCluskey method, as:

$$
\begin{aligned}
& A=\overline{x_{3}} \cdot \overline{x_{1}} \cdot x_{0} \\
& B=\overline{x_{3}} \cdot x_{1} \cdot \overline{x_{0}} \\
& C=\overline{x_{3}} \cdot x_{2} \cdot x_{0} \\
& D=\overline{x_{3}} \cdot x_{2} \cdot x_{1} \\
& E=x_{3} \cdot x_{1} \cdot \overline{x_{0}} \\
& F=\overline{x_{2}} \cdot \overline{x_{1}} \\
& G=\overline{x_{2}} \cdot \overline{x_{0}} \\
& H=x_{1} \cdot \overline{x_{0}}
\end{aligned}
$$

\begin{tabular}{|c|c|c|c|c|c|c|c|c|c|c|c|}
\hline & dec. equiv. & 0 & 1 & 2 & 5 & 6 & 7 & 8 & 9 & 10 & 14 \\
\hline$p_{i}$ & & & & & & & & & & & \\
\hline$p_{0}$ & & & 1 & & 1 & & & & & & \\
\hline$p_{1}$ & & & & 1 & & 1 & & & & & \\
\hline$p_{2}$ & & & & & 1 & & 1 & & & & \\
\hline$p_{3}$ & & & & & & 1 & 1 & & & & \\
\hline$p_{4}$ & & & & & & & & & & 1 & 1 \\
\hline$p_{5}$ & & 1 & 1 & & & & & 1 & 1 & & \\
\hline$p_{6}$ & & 1 & & 1 & & & & 1 & & 1 & \\
\hline$p_{7}$ & & & & 1 & & 1 & & & & 1 & 1 \\
\hline
\end{tabular}

Applying the Patrick method [6], going from prime implicants table will be determined all SOP solutions.

Let's consider the logical $p_{i}$ variables attached to the prime implicants as follows: $p_{0} \leftrightarrow A$, if $p_{0}=1$, the $\mathrm{A}$ prime implicant is present in the logical function expression, otherwise $p_{0}=0$ (A prime implicant is not present in the logical function expression), etc.

Therefore, considering the correspondence $p_{1} \leftrightarrow B$, $p_{2} \leftrightarrow C, p_{3} \leftrightarrow D, p_{4} \leftrightarrow E, \quad p_{5} \leftrightarrow F, \quad p_{6} \leftrightarrow G$, $p_{7} \leftrightarrow H$, in the table illustrated in Table 1 is shown the
Table 1. The SOP coverage table.

Patrick coverage:

It writes the coverage equation:

$$
\begin{aligned}
& \left(p_{5}+p_{6}\right) \cdot\left(p_{0}+p_{5}\right) \cdot\left(p_{1}+p_{6}+p_{7}\right) \\
& \cdot\left(p_{0}+p_{2}\right) \cdot\left(p_{1}+p_{3}+p_{7}\right) \cdot\left(p_{2}+p_{3}\right) \\
& \cdot\left(p_{5}+p_{6}\right) \cdot p_{5} \cdot\left(p_{4}+p_{6}+p_{7}\right) \cdot\left(p_{4}+p_{7}\right) \equiv 1
\end{aligned}
$$

Simplifications are made by using the laws of Boolean algebra: the redundance law, the identity law and the distributive law.

$$
\begin{aligned}
& p_{5} \cdot\left(p_{1}+p_{6}+p_{7}\right) \cdot\left(p_{1}+p_{3}+p_{7}\right) \\
& \cdot\left(p_{4}+p_{7}\right) \cdot\left(p_{2}+p_{0}\right) \cdot\left(p_{2}+p_{3}\right)=1 \\
& \text { or } \quad p_{5} \cdot\left(p_{7}+p_{1}+p_{3} \cdot p_{6}\right) \cdot\left(p_{7}+p_{4}\right) \\
& \quad \cdot\left(p_{2}+p_{0} \cdot p_{3}\right)=1 \\
& \text { or } \quad p_{5} \cdot\left(p_{7}+p_{1} \cdot p_{4}+p_{3} \cdot p_{4} \cdot p_{6}\right) \\
& \quad \cdot\left(p_{2}+p_{0} \cdot p_{3}\right)=1 \\
& \text { or } \quad\left(p_{5} \cdot p_{7}+p_{1} \cdot p_{4} \cdot p_{5}+p_{3} \cdot p_{4} \cdot p_{5} \cdot p_{6}\right) \\
& \quad \cdot\left(p_{2}+p_{0} \cdot p_{3}\right)=1
\end{aligned}
$$

A version of the optimal solution corresponds to $p_{5} \cdot p_{7} \cdot p_{2}$ triplet, i.e.

$$
\begin{aligned}
y & =f\left(x_{3}, x_{2}, x_{1}, x_{0}\right)=F+H+C \\
& =\overline{x_{2}} \cdot \overline{x_{1}}+x_{1} \cdot \overline{x_{0}}+\overline{x_{3}} \cdot x_{2} \cdot x_{0}
\end{aligned}
$$

The cost of this function in SOP implementation is:

$$
C(y)=C\left(\overline{x_{2}} \cdot \overline{x_{1}}\right)+C\left(x_{1} \cdot \overline{x_{0}}\right)+C\left(\overline{x_{3}} \cdot x_{2} \cdot x_{0}\right)+3=10
$$

(It was considered the variables $x_{i}, \overline{x_{i}}$, available at input).

It can verify that any other coverage has a higher cost. For example, the coverage $p_{5} \cdot p_{7} \cdot p_{0} \cdot p_{3}$ which corresponds to

$$
\begin{aligned}
y & =F+H+A+D \\
& =\overline{x_{2}} \cdot \overline{x_{1}}+x_{1} \cdot \overline{x_{0}}+\overline{x_{3}} \cdot \overline{x_{1}} \cdot x_{0}+\overline{x_{3}} \cdot x_{2} \cdot x_{1}
\end{aligned}
$$


has the cost $C_{1}(y)=14$.

\section{The Static Hazard Elimination}

(B) The consensus method

We apply the same method as in [7], only that it has a strong computing nature. Any logic function can be written as:

$$
y=e_{0} \cdot \overline{x_{i}}+e_{1} \cdot x_{i}
$$

where

$$
\begin{gathered}
e_{0}=y\left(x_{n-1}, x_{n-2}, \cdots, x_{i+1}, 0, x_{i-1}, \cdots, x_{0}\right), \\
e_{1}=y\left(x_{n-1}, x_{n-2}, \cdots, x_{i+1}, 1, x_{i-1}, \cdots, x_{0}\right) .
\end{gathered}
$$

Obviously, if $y=a \cdot \overline{x_{i}}+b \cdot x_{i}+c$ (12), then $e_{0}=a+c, e_{1}=b+c$.

If we add the term $e_{0} \cdot e_{1}$ to relation (11), the function presents no hazard towards $x_{i}$.

In terms of the consensus method, the term that covers the static 1 hazard is

$$
e_{0} \cdot e_{1}=(a+c) \cdot(b+c)=c+a \cdot b
$$

therefore for the form (11) will be $e_{0} \cdot e_{1}$, and for the form (12), $a \cdot b$.

Considering the second example, we will have: hazard in relation to the input $x_{0}$ :

$$
y=F+H+C=\overline{x_{2}} \cdot \overline{x_{1}}+x_{1} \cdot \overline{x_{0}}+\overline{x_{3}} \cdot x_{2} \cdot x_{0}
$$

where

$$
\begin{aligned}
& e_{0}=x_{1}+\overline{x_{2}} \cdot \overline{x_{1}}=x_{1}+\overline{x_{2}}, \\
& e_{1}=\overline{x_{3}} \cdot x_{2}+\overline{x_{2}} \cdot \overline{x_{1}}=\overline{x_{3}} \cdot x_{2}+\overline{x_{2}} \cdot \overline{x_{1}}, \\
& e_{0} \cdot e_{1}=\left(x_{1}+\overline{x_{2}}\right) \cdot\left(\overline{x_{3}} \cdot x_{2}+\overline{x_{2}} \cdot \overline{x_{1}}\right) \\
& =\overline{x_{3}} \cdot x_{2} \cdot x_{1}+\overline{x_{2}} \cdot \overline{x_{1}}=F+D
\end{aligned}
$$

By adding $F+D$ term to relation (14), it obtains:

$$
\begin{aligned}
y & =F+H+C+(F+D) \\
& =\overline{x_{2}} \cdot \overline{x_{1}}+x_{1} \cdot \overline{x_{0}}+\overline{x_{3}} \cdot x_{2} \cdot x_{0}+\overline{x_{3}} \cdot x_{2} \cdot x_{1}
\end{aligned}
$$

hazard in relation to the input $x_{1}$ :

$$
\begin{aligned}
& e_{0}=\overline{x_{2}}+\overline{x_{3}} \cdot x_{2} \cdot x_{0}=\overline{x_{2}}+x_{3} \cdot x_{0} \\
& e_{1}=\overline{x_{0}}+\overline{x_{3}} \cdot x_{2}+\overline{x_{3}} \cdot x_{2} \cdot x_{0}=\overline{x_{0}}+\overline{x_{3}} \cdot x_{2} \\
& e_{0} \cdot e_{1}=\left(\overline{x_{2}}+x_{3} \cdot x_{0}\right) \cdot\left(\overline{x_{0}}+\overline{x_{3}} \cdot x_{2}\right)=\overline{x_{2}} \cdot \overline{x_{0}}=G
\end{aligned}
$$

Therefore, the expression of the function becomes:

$$
\begin{aligned}
y= & F+H+C+D+G \\
= & \overline{x_{2}} \cdot \overline{x_{1}}+x_{1} \cdot \overline{x_{0}}+\overline{x_{3}} \cdot x_{2} \cdot x_{0} \\
& +\overline{x_{3}} \cdot x_{2} \cdot x_{1}+\overline{x_{2}} \cdot \overline{x_{0}}
\end{aligned}
$$

hazard in relation to the input $x_{2}$ :

$$
\begin{aligned}
& e_{0}=\overline{x_{1}}+\overline{x_{0}}+x_{1} \cdot \overline{x_{0}}=\overline{x_{1}}+\overline{x_{0}} \\
& e_{1}=\overline{x_{3}} \cdot x_{0}+\overline{x_{3}} \cdot x_{1}+x_{1} \cdot \overline{x_{0}}
\end{aligned}
$$

Therefore,

$$
\begin{aligned}
e_{0} \cdot e_{1} & =\left(\overline{x_{1}}+\overline{x_{0}}\right) \cdot\left(\overline{x_{3}} \cdot x_{0}+\overline{x_{3}} \cdot x_{1}+x_{1} \cdot \overline{x_{0}}\right) \\
& =\overline{x_{3}} \cdot \overline{x_{1}} \cdot x_{0}+\overline{x_{3}} \cdot x_{1} \cdot \overline{x_{0}}+x_{1} \cdot \overline{x_{0}} \\
& =x_{1} \cdot \overline{x_{0}}+\overline{x_{3}} \cdot \overline{x_{1}} \cdot x_{0}=H+A
\end{aligned}
$$

The expression of the function becomes:

$$
\begin{aligned}
y= & F+H+C+D+G+A \\
= & \overline{x_{2}} \cdot \overline{x_{1}}+x_{1} \cdot \overline{x_{0}}+\overline{x_{3}} \cdot x_{2} \cdot x_{0} \\
& +\overline{x_{3}} \cdot x_{2} \cdot \overline{x_{1}}+\overline{x_{2}} \cdot \overline{x_{0}}+\overline{x_{3}} \cdot \overline{x_{1}} \cdot x_{0} \\
= & \overline{x_{3}} \cdot x_{2} \cdot x_{0}+\overline{x_{3}} \cdot x_{2} \cdot \overline{x_{1}}+\overline{x_{1}} \cdot x_{0}+t
\end{aligned}
$$

hazard in relation to the input $x_{3}$ :

$$
e_{0}=x_{2} \cdot x_{0}+x_{2} \cdot \overline{x_{1}}+\overline{x_{1}} \cdot x_{0}+t,
$$

where

$$
\begin{gathered}
t=\overline{x_{2}} \cdot \overline{x_{1}}+x_{1} \cdot \overline{x_{0}}+\overline{x_{2}} \cdot \overline{x_{0}}+\overline{x_{3}} \cdot \overline{x_{1}} \cdot x_{0} \\
e_{1}=t \\
e_{0} \cdot e_{1}=\left(x_{2} \cdot x_{0}+x_{2} \cdot \overline{x_{1}}+\overline{x_{1}} \cdot x_{0}+t\right) \cdot t=t
\end{gathered}
$$

so that remains the same expression (20), which has no hazards in relation to $x_{3}$.

From the relation (20), it sees that the expression of the function without SOP hazards contains all prime implicants without $\mathrm{B}$ and $\mathrm{E}$. [8]

(C) The method of solving of some Boolean equations

A logic function can be written as:

$$
y=a \cdot \overline{x_{i}}+b \cdot x_{i}+c
$$

where

$$
\begin{aligned}
& a+c=f\left(x_{n-1}, x_{n-2}, \cdots, x_{i+1}, 0, x_{i-1}, \cdots, x_{0}\right), \\
& b+c=f\left(x_{n-1}, x_{n-2}, \cdots, x_{i+1}, 1, x_{i-1}, \cdots, x_{0}\right) .
\end{aligned}
$$

According to a theorem from [8], a logic function expressed as SOP, presents a static hazard in the situation $x_{i}+\overline{x_{i}}=1$, a situation deducted by solving the following system of logical equations:

$$
\begin{aligned}
& a=1 \\
& b=1 \\
& c=0
\end{aligned}
$$

We return to the same function, (14):

$$
y=F+H+C=\overline{x_{2}} \cdot \overline{x_{1}}+x_{1} \cdot \overline{x_{0}}+\overline{x_{3}} \cdot x_{2} \cdot x_{0} .
$$

hazard in relation to the input $x_{0}$ :

$$
y=\left(x_{1}\right) \cdot \overline{x_{0}}+\left(\overline{x_{3}} \cdot x_{2}\right) \cdot x_{0}+\overline{x_{2}} \cdot \overline{x_{1}}
$$


The function will present SOP hazard, if

$$
\begin{aligned}
& x_{1}=1 \\
& \overline{x_{3}} \cdot x_{2}=1 \\
& \overline{x_{2}} \cdot \overline{x_{1}}=0
\end{aligned}
$$

Therefore, $x_{3}=0, x_{2}=1, x_{1}=1$, which imposes a hazard at commutation

$$
\begin{aligned}
& x_{3} x_{2} x_{1} x_{0} \\
& 0110 \leftrightarrow 0111,
\end{aligned}
$$

which imposes the adding of the prime implicant $D=(6,7)$ to function.

The function becomes

$$
\begin{aligned}
y & =F+H+C+D \\
& =\overline{x_{2}} \cdot \overline{x_{1}}+x_{1} \cdot \overline{x_{0}}+\overline{x_{3}} \cdot x_{2} \cdot x_{0}+\overline{x_{3}} \cdot x_{2} \cdot x_{1}
\end{aligned}
$$

hazard in relation to the input $x_{1}$ :

$$
\begin{aligned}
& a=\overline{x_{2}}=1 \\
& x_{2}=0 \\
& b=\overline{x_{0}}+\overline{x_{3}} \cdot x_{2}=1 \\
& \text { or } x_{0}=0 \\
& c=\overline{x_{3}} \cdot x_{2} \cdot x_{0}=0 \\
& x_{3}=\phi
\end{aligned}
$$

Therefore, we will have hazards in the following cases:

$$
\begin{aligned}
& x_{3} x_{2} x_{1} x_{0} \\
& 0000 \leftrightarrow 0010 \quad(0,2) \\
& 1000 \leftrightarrow 1010 \quad(8,10)
\end{aligned}
$$

The previous commutations are equivalent to the implicant $G=(0,2,8,10)$.

The function becomes

$$
\begin{aligned}
y= & F+H+C+D+G \\
= & \overline{x_{2}} \cdot \overline{x_{1}}+x_{1} \cdot \overline{x_{0}}+\overline{x_{3}} \cdot x_{2} \cdot x_{0} \\
& +\overline{x_{3}} \cdot x_{2} \cdot x_{1}+\overline{x_{2}} \cdot \overline{x_{0}}
\end{aligned}
$$

hazard in relation to the input $x_{2}$ :

$$
\begin{aligned}
& a=\overline{x_{1}}+\overline{x_{0}}=1 \\
& x_{3}=0 \\
& b=\overline{x_{3}} \cdot x_{0}+\overline{x_{3}} \cdot x_{1}=1 \\
& \Rightarrow \text { and } c=x_{1} \cdot \overline{x_{0}}=0, x_{0}+x_{1}=1
\end{aligned}
$$

We will have the solution:

$$
\begin{aligned}
& x_{3}=0 \\
& x_{1}=0 \\
& x_{0}=1
\end{aligned}
$$

The corresponding commutation is:

$$
\begin{aligned}
& x_{3} X_{2} x_{1} x_{0} \\
& 0001 \leftrightarrow 0101 \quad(1,5) .
\end{aligned}
$$

Therefore, the term $A=(1,5)$ is added to the function.

And therefore:

$$
y=F+H+C+D+G+A
$$

hazard in relation to the input $x_{3}$ :

$$
\begin{aligned}
y= & \overline{x_{2}} \cdot \overline{x_{1}}+x_{1} \cdot \overline{x_{0}}+\overline{x_{3}} \cdot x_{2} \cdot x_{0} \\
& +\overline{x_{3}} \cdot x_{2} \cdot x_{1}+\overline{x_{2}} \cdot \overline{x_{0}}+\overline{x_{3}} \cdot \overline{x_{1}} \cdot x_{0} \\
& a=x_{2} \cdot x_{0}+x_{2} \cdot x_{1}+\overline{x_{1}} \cdot x_{0}=1 \\
& b=0 \\
& c=\overline{x_{2}} \cdot \overline{x_{1}}+x_{1} \cdot \overline{x_{0}}+\overline{x_{2}} \cdot \overline{x_{0}}=0
\end{aligned}
$$

Because one of the terms $(a, b)$ is zero, we have no hazards in relation to that variable.

\section{Conclusions}

The contribution of the authors consists in that by analysis of two methods of detection/elimination of the static hazard, insisting of the POS method for the logic function which wasn't analyzed in [1].

The boolean equation [2,3], presents some advantages instead the consensus methods, the most important to determine the transactions which causes static hazard.

It concludes that the classical method of the 70s, the method of solving some specific Boolean equations [4], presents some advantages compared to consensus method [5], which has a strong heuristic nature.

In the first method it used the consensus theorem to determine the cover term that is equal with the product of the two residual implicants [6], and in the second method it resolved a Boolean equation system [7]. The authors observed that in the second method the digital hazard can be earlier detected. If the Boolean equation system is incompatible (doesn't have solutions), the considered logical function doesn't have the static 1 hazard regarding the coupled variable. Using the logical computations, this method permits to determine the needed transitions to eliminate the digital hazard.

From the both methods, we can observe that static 1 hazard can be removed by adding the prime implicants step by step.

The same method with the same conclusions was applied to the static 0 hazard (POS), using the duality theorem $[8,9]$.

The authors observed that in the second method the digital hazard can be earlier detected. If the Boolean equation system is incompatible (doesn't have solutions), the considered logical function doesn't have the static 1 hazard regarding the coupled variable. Using the logical com- 
putations, this method permits to determine the needed transitions to eliminate the digital hazard.

\section{REFERENCES}

[1] “The Comparative Study of Two Analytical Methods for Detection and Elimination of the Static Hazard in Combinational Logic Circuits,” 2011 15th International Conference on System Theory, Control and Computing (ICSTCC), Sinaia, 14-16 Octomber 2011, pp. 1-4.

[2] R. F. Tinder, “Engineering Digital Design,” Academic Press, Waltham, 2010.

[3] Ch. Roth, "Fundamentals of Logic Design,” West Publishing Company, Eagan, 1999.

[4] J. P. Perrin, M. Denouette and E. Daclin, "Systems Lo- giques,” Tome 1 Dunord, Paris, 1997.

[5] E. T. Ringkjob, “A Method for Detection and Elimination of Static Hazards in Factored Combinational Switching Circuits,” Syracuse University, New York, 2013.

[6] J. A. McCormick, "Detection and Elimination of Static Hazards in Multilevel XOR-SOP/EQV-POS Functions,” Washington State University, Pullman, 2013.

[7] K. Raj, "Digital Systems Principles and Design,” Pearson Education India, Upper Saddle River, 2013.

[8] Al. Valachi, R. Silion, V. Onofrei and Fl. Hoza, "Analysis, Synthesis and Verification of Digital Logic Systems," Ed. Nord-Est, Iasi, 1993.

[9] O. Ursaru and C. Aghion, "Multilevel Inverters with Imbricated Switching Cells, PWM and DPWM-Controlled," Electronics and Electrical Engineering, Kaunas, 2010. 